\title{
Machine Learning and Computer Vision for the Classification of Carbon Nanotube and Nanofiber Structures from Transmission Electron Microscopy Data
}

\author{
Thomas Matson ${ }^{1 *}$, Max Farfel ${ }^{1}$, Nathan Levin ${ }^{1}$, Elizabeth Holm ${ }^{1}$ and Chen Wang ${ }^{2}$ \\ 1. Department of Materials Science and Engineering, Carnegie Mellon University, Pittsburgh, \\ Pennsylvania, USA. \\ 2. Division of Applied Research and Technology, National Institute for Occupational Safety and Health, \\ Cincinnati, Ohio, USA. \\ ${ }^{*}$ Corresponding Author: tmatson@andrew.cmu.edu
}

With increasing use of carbon nanotubes (CNTs) and carbon nanofibers (CNFs) in commercial products, several toxicity studies have indicated that these materials may be harmful to humans when exposed via inhalation [1]. Due to complex morphological features, selective identification and quantification of airborne carbon nanotube and nanofiber particles is challenging [2]. Currently, such identification and classification of these airborne nanomaterials are conducted using transmission electron microscopy (TEM). After samples are obtained and imaged, individual particles are manually parsed from the background and classified based on aspect ratios and degree of agglomeration, among other descriptors. However, this process is highly time-intensive and requires significant technical expertise to perform the classification step [3]. We present here several machine learning approaches to classify a dataset consisting of over five thousand TEM images of carbon nanotubes and nanofibers (see Figure 1), demonstrating the potential to reduce future costs and improve accuracies in manual classification.

The primary approach used in this work is transfer learning, a method in which pre-trained neural networks are used to extract features from images, which can then be used in a separate classification step. Here, we investigate three different transfer learning approaches. First, we used the VGG16 architecture, trained on ImageNet, and extracted features from the final fully connected layer of the network. We then used these features to train a linear SoftMax classifier with the classification scheme. Second, we used the ResNet50 architecture, trained on ImageNet, and extracted features from the final fully connected layer. These features were again used to train a linear SoftMax classifier. Lastly, we used the VGG16 architecture, trained on ImageNet, with a hyper-column representation [4]. The hyper-columns were constructed from the end of each convolutional block, prior to pooling, and were taken at 10 percent density. These hyper-columns were then used to create a VLAD (vector of locally aggregated descriptors) [5] encoding for each image, which was then used to train a gradient boosting classifier. The hyper-column model is particularly promising, because it preserves information about many different length scales within the image, compared to the standard transfer learning methods which only use information from the final layer of the network.

The total and categorical accuracies of each of these methods are summarized in Figure 2. Each of the methods presented here was able to classify the image dataset with an accuracy between 70 and 75 percent. Of the three, the VLAD-encoded hyper-columns had the best total accuracy at 74.7 percent but suffered in performance on classifying images of CNFs. In its current state, these methods are not accurate enough to provide useful assistance in classifying carbon nanotubes and nanofibers. In particular, we require that the hyper-column approach have a higher accuracy in the CNT fiber and CNT cluster categories. One potential reason for the dip in accuracy in the fiber class when using the hyper-column approach is the lower density of features being considered. Currently, work is being done to correct for this, by 
preprocessing the images to produce a mask prior to hyper-column selection. Additionally, we are constructing a hyper-column representation for the ResNet50 architecture and are in the process of more rigorously classifying the image dataset for training. The transfer learning approaches presented here demonstrate promising results expected to achieve upwards of 85 percent accuracy by future improvements [6].

\section{References:}

[1] G Oberdörster, V Castranova and B Asgharian, Journal of Toxicology and Environmental Health, Part B 18 (2015), p. 121.

[2] NIOSH in "Current Intelligence Bulletin 65: Occupational Exposure to Carbon Nanotubes and Nanofibers", Cincinnati, OH, US DHHS (NIOSH), Pub. No. 2013-145.

[3] M Birch, C Wang and J Fernback, NIOSH Manual of Analytical Methods 5 (2017).

[4] B Hariharan et al., eprint arXiv:14115752, (2014), p. arXiv:1411.5752.

[5] H J'egou et al., Proceedings of CVPR (2010) p. 3304.

[6] The authors acknowledge funding from the Carnegie Mellon University Department of Materials Science Senior Capstone Program, and the help of Professor Robert A. Heard.

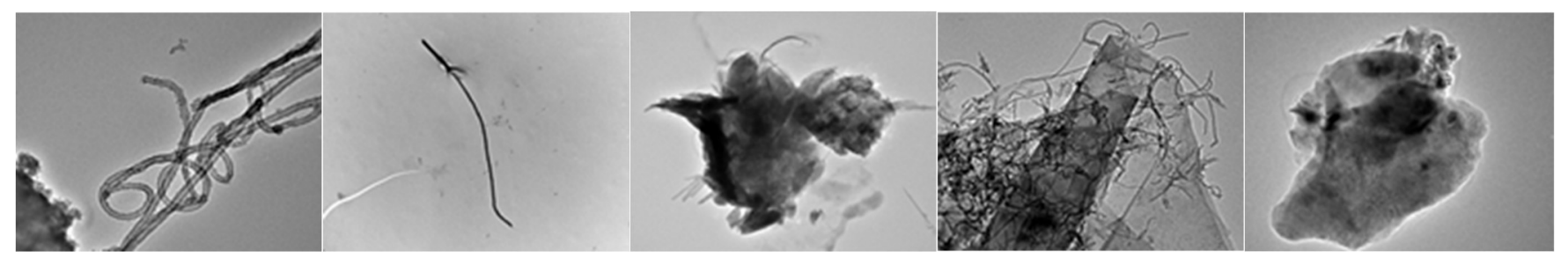

Figure 1. Example images of carbon nanostructures from the TEM image dataset. From left to right: CNT Cluster, CNT Fiber, CNT Matrix, CNT Matrix-Surface, and Non-CNT.

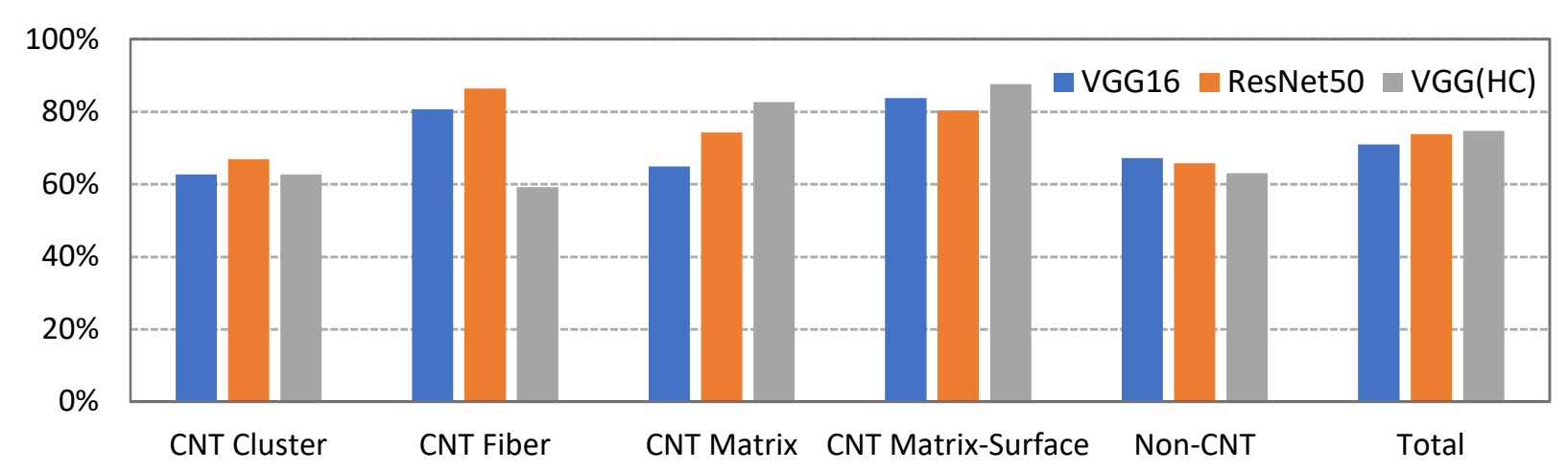

Figure 2. Categorical and total percentage accuracies for each transfer learning approach (VGG16, ResNet50, and VGG16 with VLAD-encoded hyper-columns). 